\title{
Numerical investigation of the mechanical properties of a novel hybrid polymer composite reinforced with graphene and MXene nanosheets
}

Sigitas Kilikevičius (sigitas.kilikevicius@ktu.lt), Department of Mechanical Engineering, Kaunas University of Technology, Studentu st. 56, 51424, Kaunas, Lithuania (corresponding author)

Saule Kvietkaite, Department of Mechanical Engineering, Kaunas University of Technology, Studentu st. 56, 51424, Kaunas, Lithuania

Kristina Žukiene, Department of Mechanical Engineering, Kaunas University of Technology, Studentu st. 56, 51424, Kaunas, Lithuania

Mária Omastová, Polymer Institute, Slovak Academy of Sciences, Dúbravská cesta 9, 84541 Bratislava 45, Slovakia

Andrey Aniskevich, Institute for Mechanics of Materials, University of Latvia, 23 Aizkraukles st., LV-1006, Riga, Latvia

Daiva Zeleniakiene, Department of Mechanical Engineering, Kaunas University of Technology, Studentu st. 56, 51424, Kaunas, Lithuania

\begin{abstract}
This paper presents a numerical investigation of the elastic properties of a novel hybrid polymer composite reinforced with graphene and MXene nanosheets. A finite element computational model was developed to analyze the mechanical properties of a new polymer hybrid composite reinforced with MXene and graphene taking into account the properties of the 2D nanosheets, different aspect ratios, placement options and volume fractions of nanoreinforcements, as well as the interaction effects between the nanofillers and the surrounding polymer matrix. Using the developed numerical model, the influences of the interface layer properties, MXene and graphene aspect ratio, alignment and volume fraction on the orthotropic mechanical properties of the proposed novel hybrid polymer nanocomposites were determined. The results suggest that MXenes along with graphene nanosheets show considerable promise in the development of novel nanoengineered high-strength multifunctional composite materials, as well as provides insight for future design of such materials.
\end{abstract}

Keywords: hybrid composites, MXene, graphene, numerical simulation, elastic properties.

This is the author's version of a work that was accepted for publication in the journal Computational Materials Science. Changes resulting from the publishing process, such as editing, corrections, structural formatting, and other quality control mechanisms may not be reflected in this document. A definitive version was subsequently published in the journal Computational Materials Science, Volume 174, March 2020, 109497, doi.org/10.1016/j.commatsci.2019.109497.

The final publication is available at https://www.sciencedirect.com/science/article/pii/S0927025619307967 


\section{Introduction}

Presently, graphene attracts more attention than all other 2D materials together. The main advantages of graphene are excellent conductivity and mechanical properties. Graphene is used in a wide range of applications requiring lightweight, high strength polymer composite materials [1-4]. However, the commercial use of graphene is still limited by low production, as it is expensive. Graphene has a hydrophobic surface, resulting in agglomeration, poor compatibility and dispersibility in polymers, weak interfaces and insufficient mechanical reinforcement effects [5-6]. These parameters motivate the search for new materials meeting the requirements of contemporary technology.

A new family of 2D nanomaterials was discovered in 2011, referred to as MXenes. MXenes were produced by the extraction of the A-group layers from the transition metal carbides and/or nitrides, known as the MAX phases [7]. MXenes conduct heat and electricity similarly to metals; however, they are strong, elastically stiff, brittle and heat-tolerant like ceramics $[7,8]$. Moreover, they are resistant to chemically aggressive environments, relatively easily machinable, tolerant to damage and high temperature as well as resistant to fatigue, creep and oxidation [9]. $\mathrm{Ti}_{3} \mathrm{C}_{2}$ is one of the most common MXenes, produced from $\mathrm{Ti}_{3} \mathrm{AlC}_{2}$, and exhibits outstanding performances in many applications [10-15]. It is characterized by a high in-plane Young's modulus as well as high bending rigidity and strength [16-17]. Taking these characteristics into account, MXenes show considerable promise as fillers for novel nanoengineered high-strength multifunctional composite materials; therefore, research and development of hybrid polymer composites by a combination of MXenes and graphene are currently very relevant topics. The exploration of MXenes is still in the preliminary stages, and opportunities are wide open for developing MXene reinforced composites for various practical applications.

Despite the relatively easy manufacturing technology, MXenes are produced in quite limited quantities and, consequently, are not able to completely fulfill the growing demands for their scientific and practical applications. Therefore, computational models can be very effective to estimate the mechanical properties of nanoreinforced composites and to find recommendations for optimization of the composition of these materials. Theoretical and numerical methods for investigation of nanoreinforced composites have been developed based on the combination of atomistic simulations and continuum and structural mechanics [18]. Among them, the finite element-based method is commonly used in evaluating the material properties and behavior [19-22] due to the maturity of contemporary microstructure development techniques and the advances in material modeling [23]. Nevertheless, the vast majority of research works utilizing the finite element-based approach were focused on graphene for improving the mechanical properties of nanocomposites [24-26]. Recent studies on effective elasticity properties using numerical methods aimed to determine the anisotropic 3D elastic behavior of diamond-based composites [27], investigate transversely isotropic properties of carbon nanotube (CNT)-polymer composites [28], analyze elastic properties of laminate composites reinforced with thin films composed of CNTs for military applications [29], predict elastic properties of a polymer hybrid composite with unidirectional carbon fibers coated with randomly oriented CNTs [30], and study the elastic response of bioinspired composite materials with high volume fractions of hexagonal and cylindrical inclusions [31]. Recent studies demonstrated great improvements in the mechanical properties of composites resulting from sophisticated chosen reinforcements, which motivates the search for new compositions of materials and their applications.

The aim of this study is to analyze the mechanical properties of a new hybrid polymer composite reinforced with MXene and graphene 2D nanosheets by developing a finite element computational model, which would give the first insight on the proposed sort of composites, encourage research and facilitate practical applications, as well as provide insight for future design of similar composite materials. 


\section{Computational model}

To investigate the elastic mechanical properties of the new hybrid polymer composite reinforced with MXene and graphene 2D nanosheets, a series of three dimensional computational microstructural models, which are called representative volume elements (RVEs), were developed. The RVEs were modeled with Digimat-FE (Extreme Engineering, MSC Software, Belgium) using various aspect ratios of graphene $\rho_{G}$ and MXene $\rho_{M X}$ inclusions (the diameter-to-thickness ratio), as well as various alignments and volume fractions $\left(f_{G}\right.$ and $f_{M X}$ ). In the RVEs with randomly oriented inclusions, the volume fraction of graphene was $0.05 \%$, while the volume fraction of MXene was changed from $0.05 \%$ to $0.5 \%$. In the RVEs with aligned inclusions, the volume fraction of graphene was $0.2 \%$, while the volume fraction of MXene was changed from $0.2 \%$ to $1.4 \%$. An aspect ratio value of 1194 was used for the graphene inclusions, while aspect ratio values of 200 and 400 were used for the MXene inclusions.

The graphene inclusions were modeled as discs with a thickness of $0.335 \mathrm{~nm}$ [26], while the MXenes were modeled as discs with a thickness of $1 \mathrm{~nm}[7,9,32]$. It is known that nanofiller/polymer matrix interfaces influence the mechanical properties of nanocomposites. The thickness of the effective interface between inclusions and matrix was set to $1 \mathrm{~nm}$ based on the research [26]. The size of RVEs with randomly aligned inclusions was set to the diameter of the biggest inclusion multiplied by 3.5 times. For the RVEs with aligned inclusions, the edge sizes along the alignment direction was 3.5 times the diameter of the biggest inclusion and the edge size perpendicular to the alignment direction (along the 2-direction) was 0.2 times the edge size along the alignment direction. Figure 1 shows an RVE with randomly oriented inclusions as $\left(\rho_{G}=1194, \rho_{M X}=200, f_{G}=0.05 \%\right.$, and $\left.f_{M X}=0.5 \%\right)$ (a) along with an RVE with aligned inclusions $\left(\rho_{G}=1194, \rho_{M X}=400, f_{G}=0.2 \%\right.$, and $\left.f_{M X}=1.4 \%\right)(\mathrm{b})$. The MXene inclusions coated with the effective interface are colored in green, while the graphene inclusions coated with the effective interface are colored in blue.

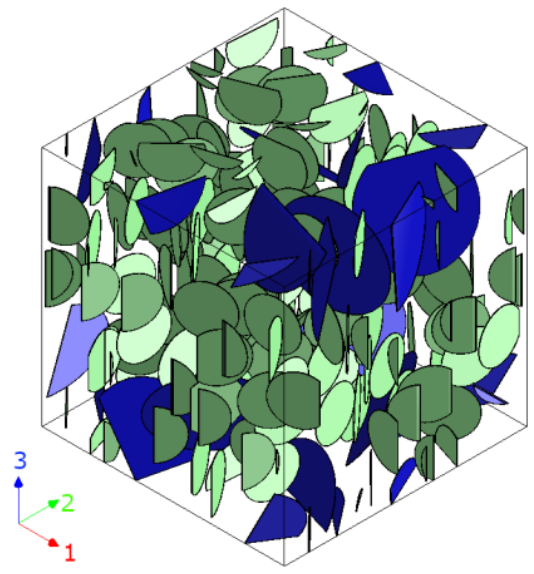

(a)

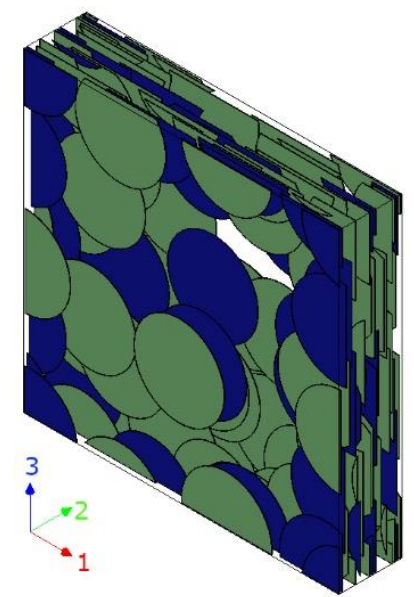

(b)

Figure 1. Examples of modeled RVEs: with randomly orientated inclusions ( $\rho_{\mathrm{G}}=1194$, $\rho_{M X}=200, f_{\mathrm{G}}=0.05 \%$, and $\left.f_{M X}=0.5 \%\right)$ (a) and with aligned inclusions $\left(\rho_{G}=1194 ; \rho_{M X}=400\right.$,

$$
f_{G}=0.2 \% \text {, and } f_{M X}=1.4 \% \text { ) (b) }
$$

The isotropic linear elastic material model was applied in this study to describe the behaviors of inclusion, interface and matrix materials, and it was assumed that the inclusions are perfectly bonded to the interface layers and that the interface layers are perfectly bonded to the matrix. This approach is suitable to determine the elastic behavior of the RVEs [24, 31]. Moreover, regarding the interface between MXenes and epoxy, this assumption is also based on the initial researches on surface energies of MXene and its interfacial adhesion energies with epoxy, as well as the analysis of scanning electron microscopy images of fractured surfaces of 
epoxy/MXene/graphene composites demonstrates good adhesion properties. The mechanical properties of the graphene-matrix interphase were based on the research results obtained using the inverse modeling approach, as presented in [26]. The mechanical properties of the materials used in the model are based on the analysis of literature data and are presented in Table 1.

Table 1. The mechanical properties of the materials

\begin{tabular}{lcccc}
\hline \multirow{2}{*}{ Material property } & \multicolumn{4}{c}{ Materials } \\
\cline { 2 - 5 } & $\begin{array}{c}\text { MXene (Ti3C2) } \\
{[34]}\end{array}$ & $\begin{array}{c}\text { Graphene } \\
{[33]}\end{array}$ & $\begin{array}{c}\text { Effective } \\
\text { interface [26] }\end{array}$ & Epoxy [25] \\
\hline Young's modulus, GPa & 330 & 1000 & 3.74 & 2.5 \\
Poisson's ratio & 0.23 & 0.165 & 0.35 & 0.35 \\
\hline
\end{tabular}

Three-dimensional periodic boundary conditions (PBCs) were applied to represent an infinite material domain [31], and the models were subjected to normal and pure shear loading cases in order to determine anisotropic mechanical properties of the composite. The applied strain for normal and pure shear loading was 0.005 .

For the inhomogeneous materials, the relation between volume averaged stress and volume averaged strain is determined as follows [35]:

$$
\begin{gathered}
\left\langle\varepsilon_{i j}\right\rangle=\frac{1}{\left|\varphi_{e}\right|} \int_{\varphi} \varepsilon_{i j}^{0}(x, y) d \varphi_{e} \\
\left\langle\sigma_{i j}\right\rangle=\frac{1}{\left|\varphi_{e}\right|} \int_{\varphi} \sigma_{i j}^{0}(x, y) d \varphi_{e}=C_{i j k l}^{H}\left(\varepsilon^{0}\right)\left\langle\varepsilon_{k l}\right\rangle
\end{gathered}
$$

where $\left\langle\varepsilon_{i j}\right\rangle$ and $\left\langle\sigma_{i j}\right\rangle$ are the volume averaged strain and stress, respectively; $\varepsilon_{i j}^{0}$ and $\sigma_{i j}^{0}$ are the local strain and stress, respectively; $\varphi_{e}$ is the total volume of the RVE; $C_{i j k l}^{H}$ is the equivalent homogenized stiffness matrix. $C_{i j k l}^{H}$ constants can be calculated by applying six macrostrains and PBCs for six independent models. The RVE models were meshed using tetrahedral elements (the meshes consisted of $\sim 3-4$ million elements depending on the number of inclusions) and the volume averaged stresses and strains were calculated numerically by solving the FEM models using the commercial finite element software ANSYS (ANSYS, Inc., USA.).

The effective stiffness matrix can be calculated as follows:

$$
\left[\begin{array}{l}
\sigma_{11} \\
\sigma_{22} \\
\sigma_{33} \\
\tau_{23} \\
\tau_{13} \\
\tau_{12}
\end{array}\right]=\left[\begin{array}{cccccc}
C_{11} & C_{12} & C_{13} & 0 & 0 & 0 \\
C_{12} & C_{22} & C_{26} & 0 & 0 & 0 \\
C_{13} & C_{23} & C_{33} & 0 & 0 & 0 \\
0 & 0 & 0 & C_{44} & 0 & 0 \\
0 & 0 & 0 & 0 & C_{55} & 0 \\
0 & 0 & 0 & 0 & 0 & C_{66}
\end{array}\right]\left[\begin{array}{l}
\varepsilon_{11} \\
\varepsilon_{22} \\
\varepsilon_{33} \\
\gamma_{23} \\
\gamma_{13} \\
\gamma_{12}
\end{array}\right]
$$

The inverted form of the $C_{i j k l}^{H}$ matrix is the compliance matrix $S_{i j k l}^{H}$. The elastic constants can be calculated using the following [31]: 


$$
[S]=\left[\begin{array}{cccccc}
\frac{1}{E_{1}} & \frac{-v_{21}}{E_{2}} & \frac{-v_{31}}{E_{3}} & 0 & 0 & 0 \\
\frac{-v_{21}}{E_{2}} & \frac{1}{E_{2}} & \frac{-v_{32}}{E_{3}} & 0 & 0 & 0 \\
\frac{-v_{31}}{E_{3}} & \frac{-v_{31}}{E_{3}} & \frac{1}{E_{3}} & 0 & 0 & 0 \\
0 & 0 & 0 & \frac{1}{G_{23}} & 0 & 0 \\
0 & 0 & 0 & 0 & \frac{1}{G_{13}} & 0 \\
0 & 0 & 0 & 0 & 0 & \frac{1}{G_{12}}
\end{array}\right]
$$

\section{Simulation results}

As there are no data on the mechanical properties of the effective interface between MXenes and epoxy matrix, a simulation was carried out on the aligned RVE with $\rho_{G}=1194$, $\rho_{M X}=400, f_{G}=0.2 \%$, and $f_{M X}=1.4 \%$ to determine the influence of its Young's modulus $E_{M X}^{i}$ on the effective mechanical properties of the RVE. The results showed that the normalized effective moduli $\bar{E}_{1} / E_{m}$ and $\bar{E}_{2} / E_{m}$ increase nonlinearly as $E_{M X}^{i}$ increases (Figure 2 (a)). However, this increase is not very significant. Additionally, some slight changes in the effective Poisson's ratios were observed as well (Figure 2 (b)). In this way, the Young modulus value of the MXene-matrix interface of $3.74 \mathrm{GPa}$ was used in the following simulations.

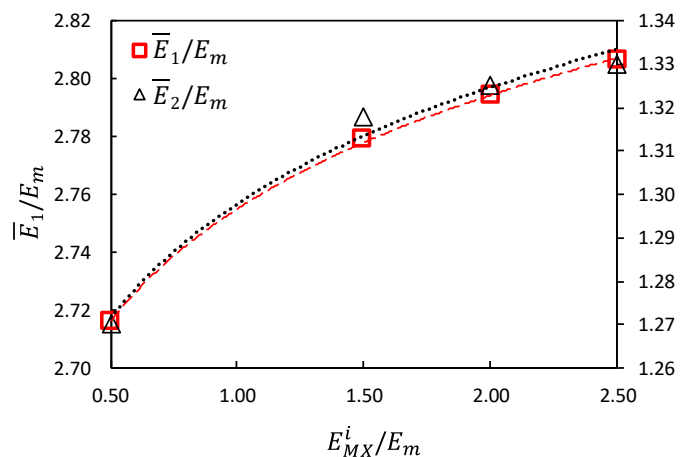

(a)

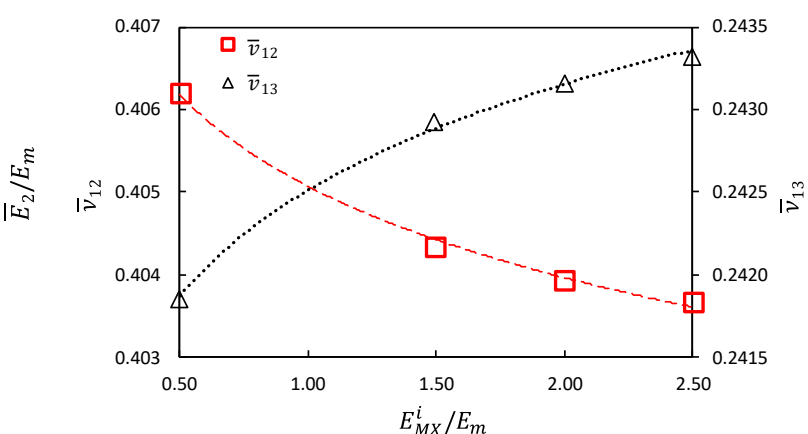

(b)

Figure 2. The influence of the effective interface on the normalized effective elastic moduli (a) and Poisson's ratios (b)

Typical normal stress $\sigma_{1}$ contours under uniaxial tension in the 1-direction, and shear stress $\tau_{12}$ contours under pure shear applied in the 12-plane for the RVE with randomly distributed inclusions $\left(\rho_{G}=1194, \rho_{M X}=200, f_{G}=0.05 \%\right.$, and $\left.f_{M X}=0.5 \%\right)$ are presented in Figure 3 and Figure 4, respectively. The matrix without inclusions and interface coatings is shown in Figure 3 (a) and Figure 4 (a), while the inclusions are shown in Figure 3 (b) and Figure 4 (b). The highest magnitudes of the normal stress $\sigma_{1}$ and shear stress $\tau_{12}$ were observed in graphene inclusions. 


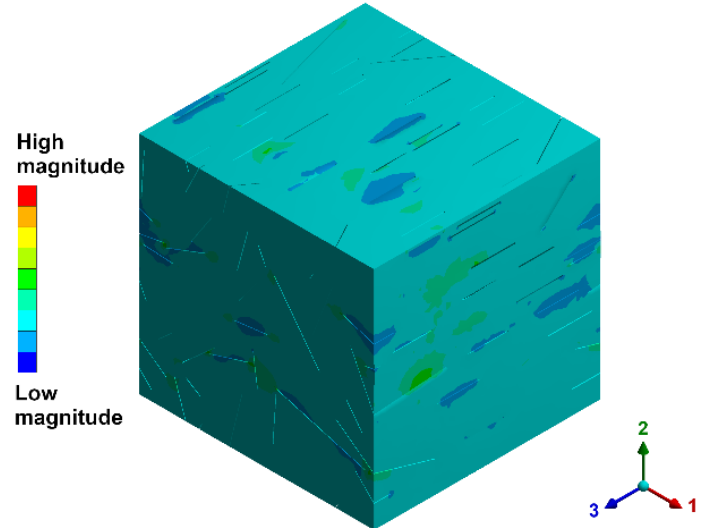

(a)

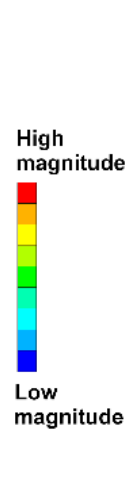

Figure 3. Normal stress $\sigma_{1}$ contours for the RVE with randomly distributed inclusions ( $\rho_{G}=1194, \rho_{M X}=200, f_{G}=0.05 \%$, and $f_{M X}=0.5 \%$ ) under uniaxial tension in the 1-direction: the

matrix (a); the graphene and MXene inclusions (b)

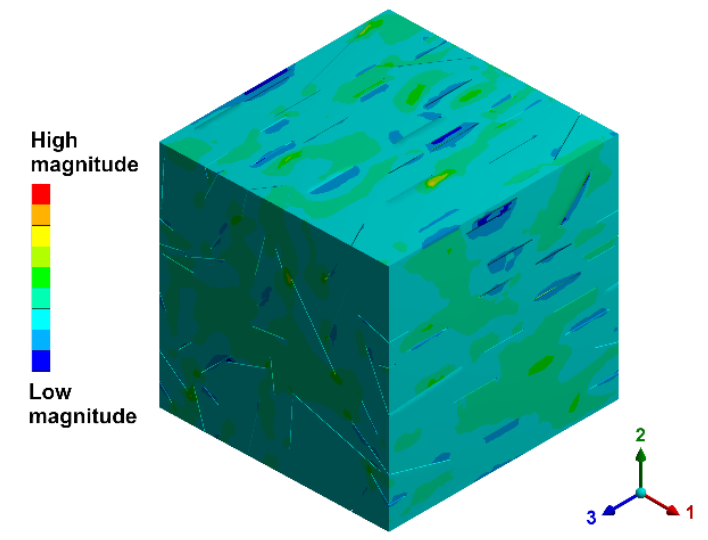

(a)

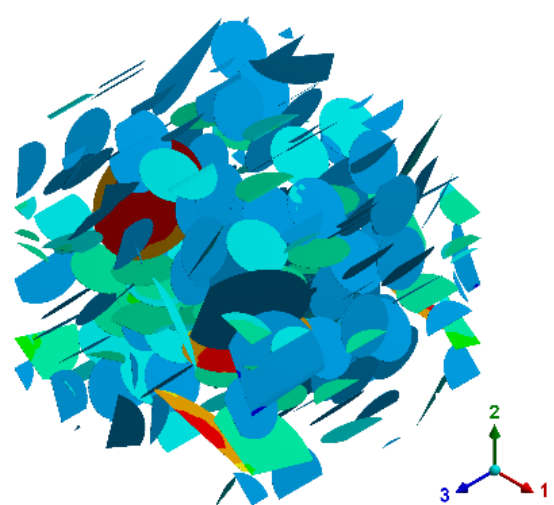

(b)

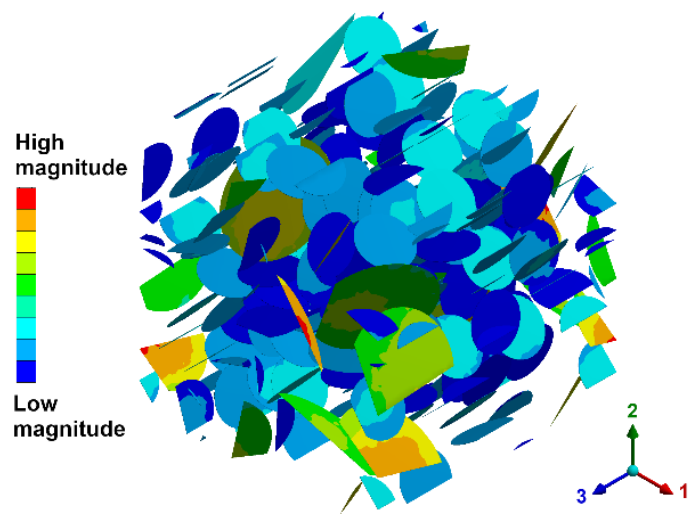

(b)

Figure 4. Shear stress $\tau_{12}$ contours for the RVE with randomly distributed inclusions ( $\rho_{G}=1194, \rho_{M X}=200, f_{G}=0.05 \%$, and $f_{M X}=0.5 \%$ ) under pure shear applied in the 12-plane: the matrix (a); the graphene and MXene inclusions (b)

Normal stress $\sigma_{1}$ contours under uniaxial tension in the 1-direction and shear stress $\tau_{13}$ contours under pure shear applied in the 13-plane for the RVE with aligned inclusions $\left(\rho_{G}=1194, \rho_{M X}=400, f_{G}=0.2 \%\right.$, and $\left.f_{M X}=1.4 \%\right)$ are presented in Figure 5 and Figure 6, respectively. The matrix without the inclusions and the interface coatings is shown in Figure 5 (a) and Figure 6 (a), while the inclusions are shown in Figure 5 (b) and Figure 6 (b). The highest magnitudes of $\sigma_{1}$ and $\tau_{13}$ were observed in graphene inclusions.

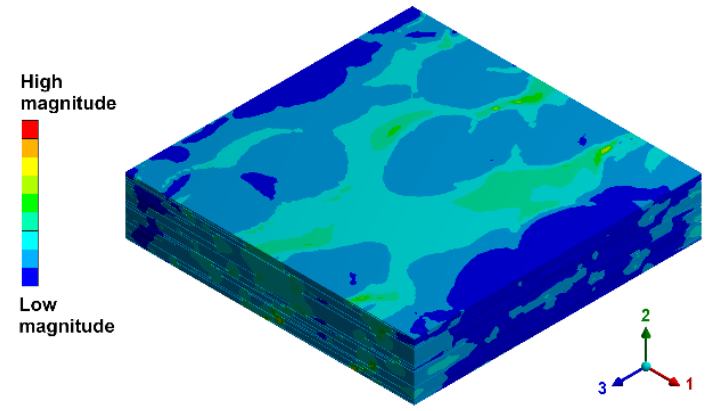

(a)

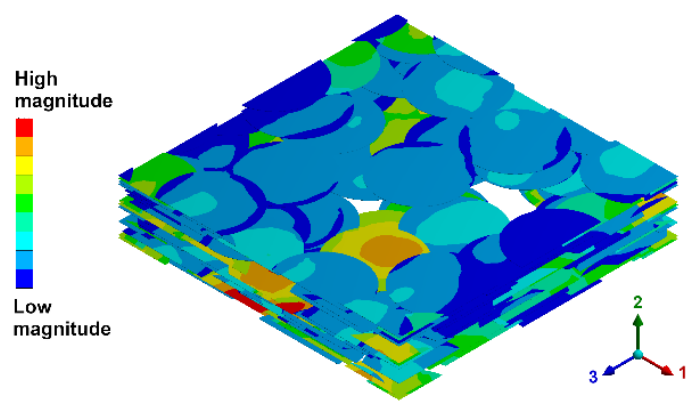

(b)

Figure 5. Normal stress $\sigma_{1}$ contours for the RVE with aligned inclusions ( $\rho_{G}=1194 ; \rho_{M X}=400$, $f_{G}=0.2 \%$, and $f_{M X}=1.4 \%$ ) under uniaxial tension in the 1-direction: the matrix (a); the graphene and MXene inclusions (b) 


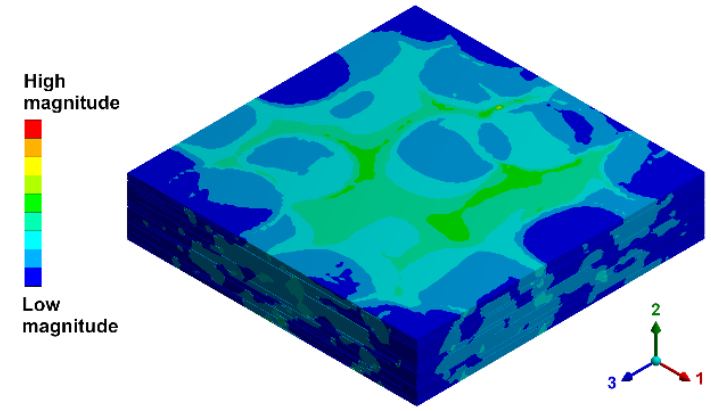

(a)

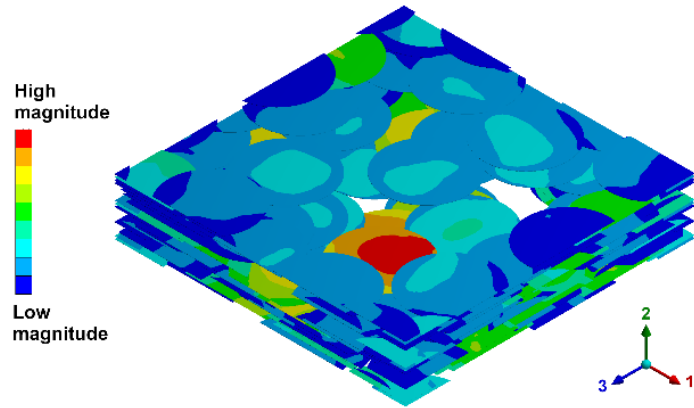

(b)

Figure 6. Shear stress $\tau_{13}$ contours for the RVE with aligned inclusions ( $\rho_{G}=1194$; $\rho_{M X}=400, f_{G}=0.2 \%$, and $f_{M X}=1.4 \%$ ) under pure shear applied in the 13-plane: the matrix (a); the graphene and MXene inclusions (b)

The simulation showed that the normalized effective elastic modulus $\bar{E} / E_{m}$ increases when the volume fraction of MXene increases in the RVEs with random placement of the inclusions (Figure 7 (a)), as does the normalized effective shear modulus $\bar{G} / G_{m}$ (Figure 7 (b)). The simulation demonstrated that $\bar{E} / E_{m}$ and $\bar{G} / G_{m}$ ere higher under higher aspect ratios of MXene.

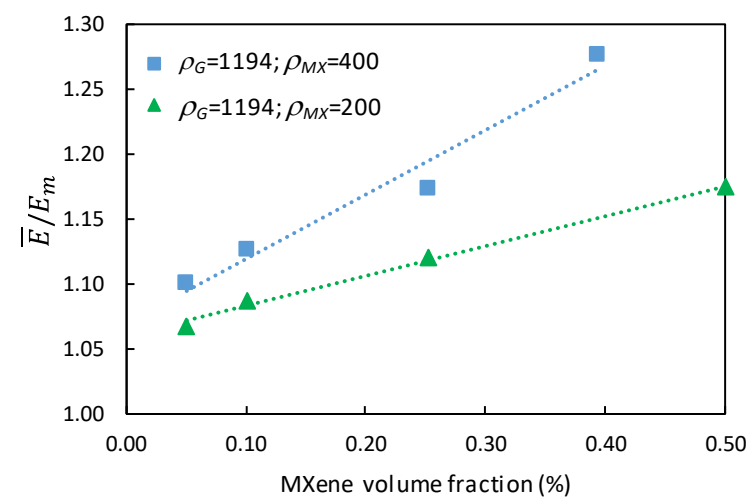

(a)

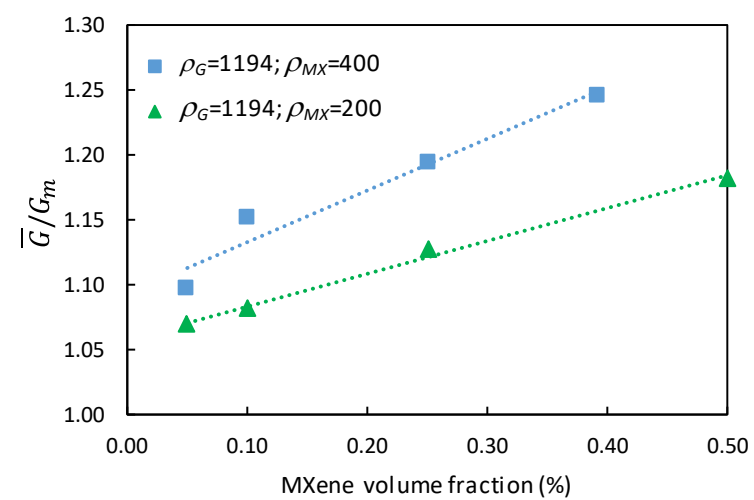

(b)

Figure 7. The mechanical properties of the RVE's with randomly distributed inclusions as $f_{G}=0.05 \%: \bar{E} / E_{m}$ (a) and $\bar{G} / G_{m}$ (b) vs MXene volume fractions under different aspect ratios of the inclusions

Orthotropic effective mechanical properties were determined for the RVEs with aligned inclusions (Figure 8-Figure 11). Both the normalized effective moduli $\bar{E}_{1} / E_{m}$ (Figure 8 (a)) and $\bar{E}_{2} / E_{m}$ (Figure $8(\mathrm{~b})$ ) increase when MXene volume fraction increases; however, $\bar{E}_{1} / E_{m}$ is significantly higher in comparison to $\bar{E}_{2} / E_{m}$. The simulation demonstrated that by changing the volume fraction of MXene from $0.2 \%$ to $1.4 \%, \bar{E}_{1} / E_{m}$ is increased from $\sim 1.7$ to $\sim 2.8$ for the aspect ratio of $\rho_{M X}=400$, as well as from $\sim 1.6$ to $\sim 2.6$ for the aspect ratio of $\rho_{M X}=200$. Similarly, $\bar{G}_{13} / G_{m}$ (Figure 8 (d)) demonstrates a more significant increase in comparison to $\bar{G}_{12} / G_{m}$ (Figure $8(\mathrm{c})$ ). By changing the volume fraction of MXene from $0.2 \%$ to $1.4 \%, \bar{G}_{13} / G_{m}$ is increased from $\sim 1.8$ to $\sim 2.8$ for the aspect ratio of $\rho_{M X}=400$, as well as from $\sim 1.7$ to $\sim 2.7$ for the aspect ratio of $\rho_{M X}=200$. Higher aspect ratios of MXene contribute to higher magnitudes of $\bar{E}_{1} / E_{m}, \bar{E}_{2} / E_{m}$. and $\bar{G}_{13} / G_{m}$. Although, lower values of $\bar{G}_{12} / G_{m}$ were observed under the higher value of the aspect ratio of MXene. 


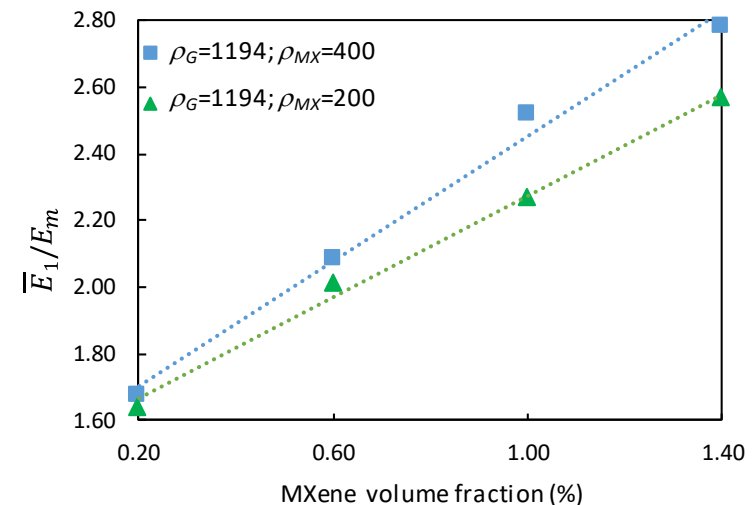

(a)

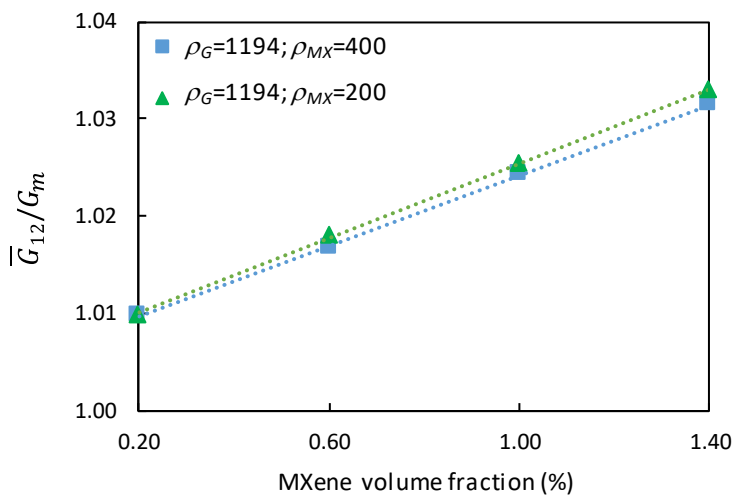

(c)

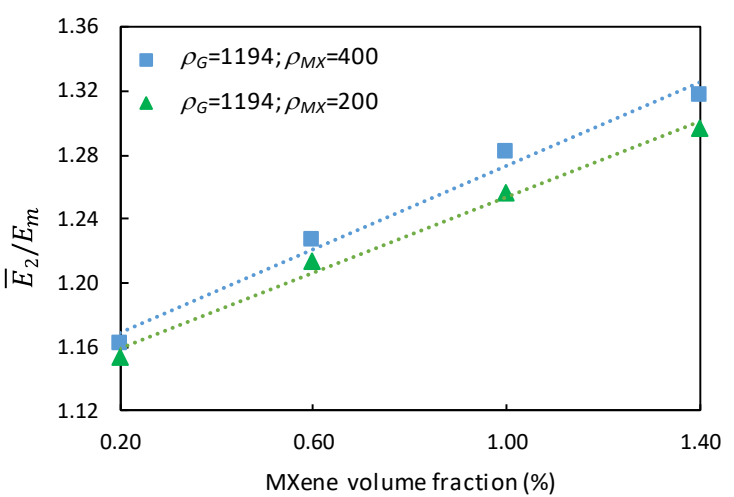

(b)

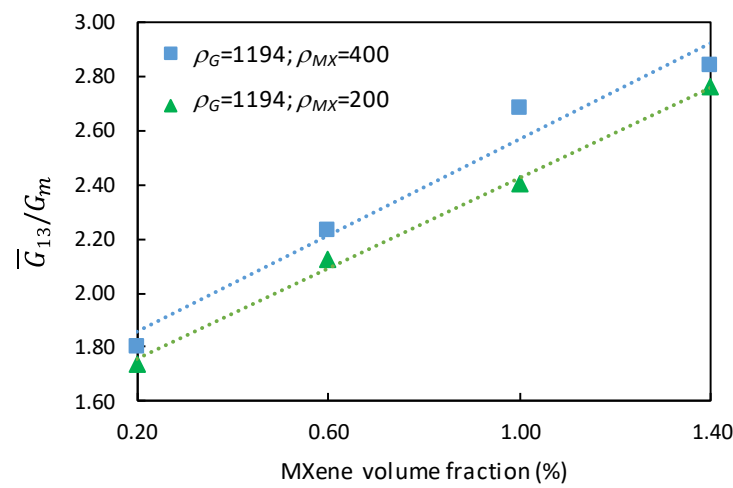

(d)

Figure 8. The mechanical properties of the RVE's with aligned inclusions as $f_{G}=0.2 \%$ : $\bar{E}_{1} / E_{m}(\mathrm{a}), \bar{E}_{2} / E_{m}$ (b), $\bar{G}_{12} / G_{m}$ (c) and $\bar{G}_{13} / G_{m}$ (d) vs MXene volume fractions under different aspect ratios of the inclusions

Figure 9 demonstrates the linear relationship between effective Poisson's ratios and MXene volume fractions in the analyzed range. As it is expected due to the inclusion alignment parallel to the 13-plane, an increase in the volume fraction of MXene from $0.2 \%$ to $1.4 \%$, leads to an increase of $\bar{v}_{12}$ from 0.385 to 0.404 for $\rho_{M X}=400$ and from 0.383 to 0.402 for $\rho_{M X}=200$. Meanwhile, $\bar{v}_{13}$ decreases from 0.284 to 0.243 for $\rho_{M X}=400$ and from 0.287 to 0.248 for $\rho_{M X}=200$, as well as $\bar{v}_{23}$ decreases from 0.259 to 0.189 for $\rho_{M X}=400$ and from 0.266 to 0.202 for $\rho_{M X}=200$.

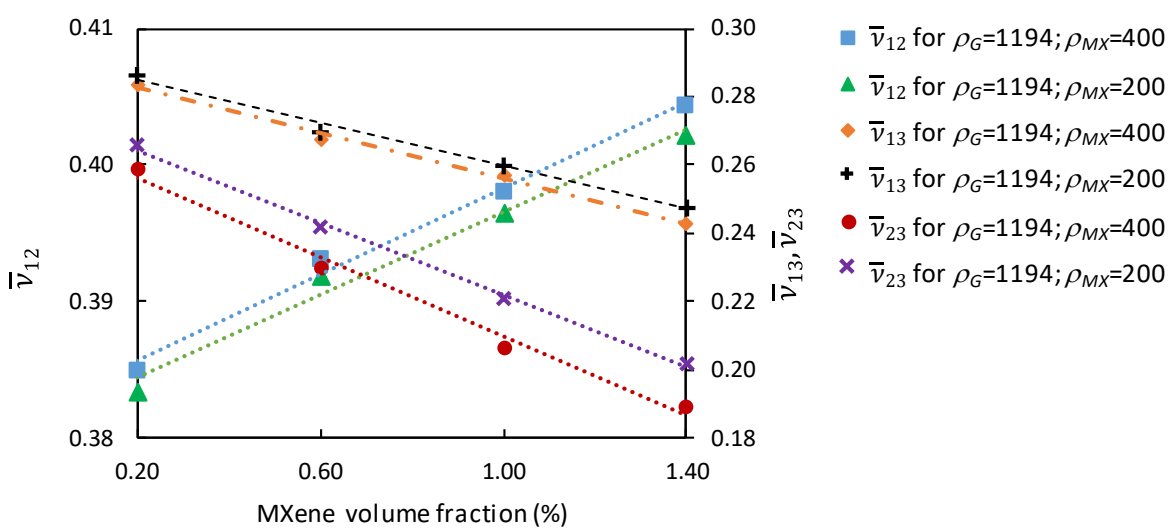

Figure 9. Effective Poisson's ratios vs MXene volume fractions under different aspect ratios of the inclusions as $f_{G}=0.2 \%$

Similar influences of the graphene volume fraction on the effective moduli (Figure 10) and Poisson's ratios (Figure 11) were observed. By increasing the volume fraction of graphene in 
the hybrid composite up to $0.3 \%$, while keeping $\bar{E}_{1} / E_{m}$ is increased from $\sim 1.8$ to $\sim 2.7$ and $\bar{G}_{13} / G_{m}$ is increased from $\sim 1.9$ to $\sim 2.9$.

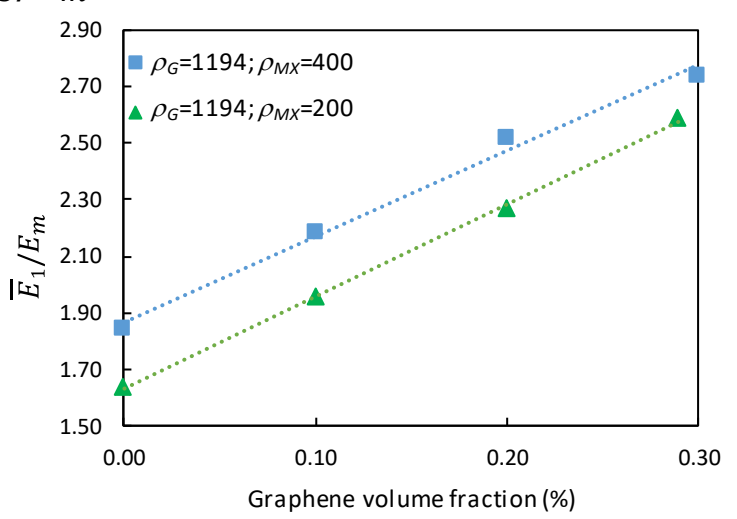

(a)

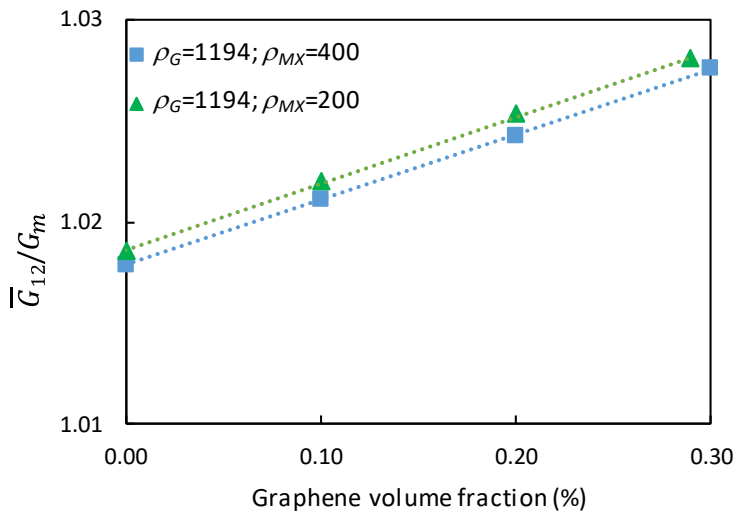

(c)

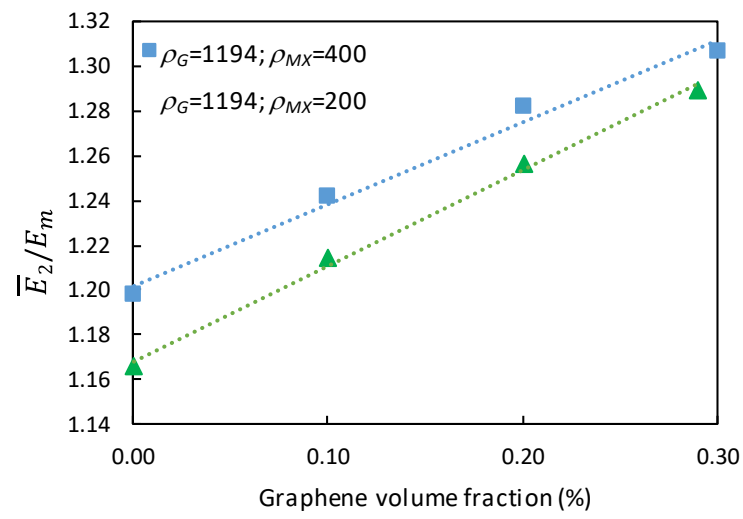

(b)

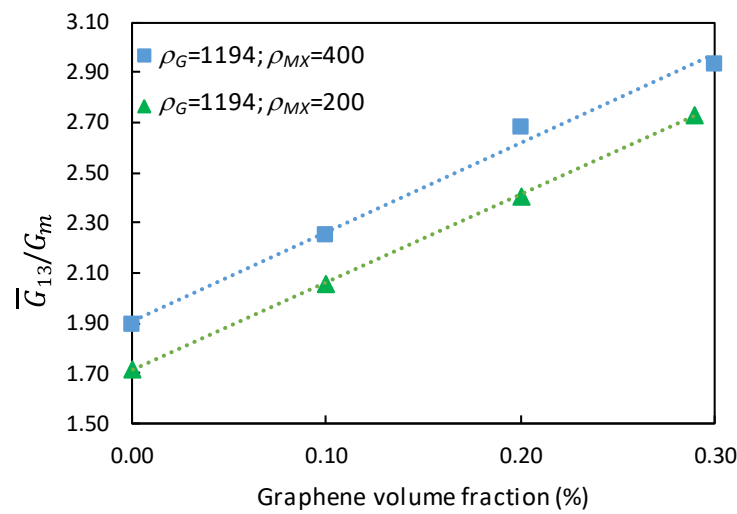

(d)

Figure 10. Mechanical properties of the RVE's with aligned inclusions as $f_{M X}=1 \%: \bar{E}_{1} / E_{m}$ (a), $\bar{E}_{2} / E_{m}(\mathrm{~b}), \bar{G}_{12} / G_{m}(\mathrm{c})$ and $\bar{G}_{13} / G_{m}(\mathrm{~d})$ vs graphene volume fractions under different aspect ratios of the inclusions

An increase in the volume fraction of graphene in the hybrid composite up to $0.3 \%$, leads to an increase of $\bar{v}_{12}$ from 0.383 to 0.402 for $\rho_{M X}=400$ and from 0.375 to 0.406 for $\rho_{M X}=200$. Meanwhile, $\bar{v}_{13}$ decreases from 0.285 to 0.249 for $\rho_{M X}=400$ and from 0.301 to 0.242 for $\rho_{M X}=200$, as well as $\bar{v}_{23}$ decreases from 0.247 to 0.195 for $\rho_{M X}=400$ and from 0.267 to 0.204 for $\rho_{M X}=200$.

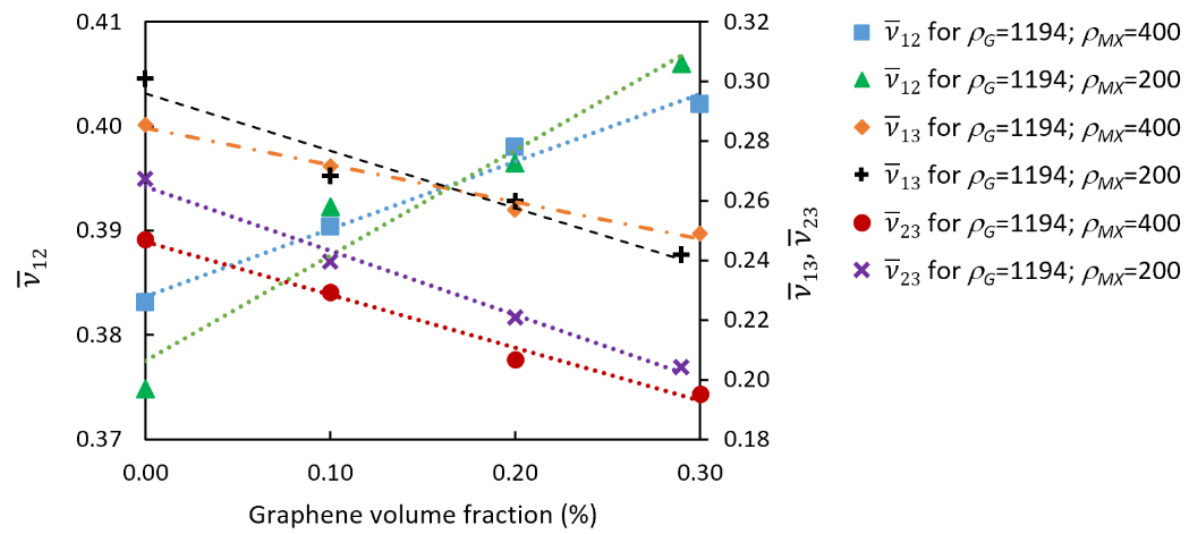

Figure 11. Effective Poisson's ratios vs graphene volume fraction under different aspect ratios of the inclusions as $f_{M X}=1 \%$ 


\section{Conclusions}

A finite element computational model was developed to analyze the mechanical properties of a new polymer composite reinforced with MXene and graphene 2D nanosheets taking into account the properties of the 2D nanosheets, different aspect ratios, placement options and volume fractions of nanoreinforcements, as well as the interaction effects between the nanofillers and the surrounding polymer matrix.

The simulation results showed that MXenes significantly increase the elastic properties of the composite. The simulation results showed that the normalized effective elastic moduli increase when the volume fractions of MXene increase. All of these trends were observed in both types of RVEs with random and aligned placement of the inclusions. However, the aligned placement of the inclusion leads to significantly higher stiffness along the alignment direction. Higher aspect ratios also contribute to a higher increase in stiffness. A similar influence of the graphene volume fraction on the mechanical properties of the hybrid composite was also observed. Despite the fact that graphene increases the stiffness of the composite more significantly in comparison to MXene, it is expected that MXenes will expand the scope of applications of graphene reinforced hybrid nanocomposites as the addition of MXenes might contribute to a reduction of costs, a simplification of manufacturing processes, an enhancement of electrical transport, etc. The results suggest that MXenes along with graphene nanosheets show considerable promise in the development of novel nanoengineered high-strength multifunctional composite materials, as well as provides insight for future design of such materials.

\section{Data availability}

The raw/processed data will be made available on reasonable request.

\section{Acknowledgements}

This work was developed under the M-era.Net research project titled NANO2COM Advanced Polymer Composites Filled with Novel 2D Nanoparticles. The research was funded by the following grants: No. S-M-ERA.NET-18-1 from the Research Council of Lithuania, No. 1.1.1.5/ERANET/18/02 from the Latvian State Education Development Agency, and No. MERANET-18-414-Nano2Com from the Slovak Academy of Sciences.

\section{References}

1. Ma, X., Shen, B., Zhang, L., Liu, Y., Zhai, W. and Zheng, W. Porous superhydrophobic polymer/carbon composites for lightweight and self-cleaning EMI shielding application. Composites Science and Technology, 158, 86-93 (2018).

2. She, X., He, C., Peng, Z. and Kong, L. Molecular-level dispersion of graphene into epoxidized natural rubber: Morphology, interfacial interaction and mechanical reinforcement. Polymer, 55(26), 6803-6810 (2014).

3. Mittal, G., Rhee, K. Y., Mišković-Stanković, V. and Hui, D. Reinforcements in multi-scale polymer composites: processing, properties, and applications. Composites Part B: Engineering, 138, 122-139 (2018).

4. Shi, G., Araby, S., Gibson, C. T., Meng, Q., Zhu, S. and Ma, J. Graphene platelets and their polymer composites: fabrication, structure, properties, and applications. Advanced Functional Materials, 28(19), 1706705 (2018).

5. Prolongo, S. G., Moriche, R., Jiménez-Suárez, A., Sánchez, M. and Ureña, A. Advantages and disadvantages of the addition of graphene nanoplatelets to epoxy resins. European Polymer Journal, 61, 206-214 (2014). 
6. Starkova, O., et al. Hydrothermally resistant thermally reduced graphene oxide and multiwall carbon nanotube based epoxy nanocomposites. Polymer Degradation and Stability, 98(2), 519-526 (2013).

7. Naguib, M., et al. Two-dimensional nanocrystals produced by exfoliation of Ti3AlC2. Advanced Materials, 23(37), 4248-4253 (2011).

8. Ling, Z., et al. Flexible and conductive MXene films and nanocomposites with high capacitance. Proceedings of the National Academy of Sciences, 111(47), 16676-16681 (2014).

9. Naguib, M., Mochalin, V. N., Barsoum, M. W. and Gogotsi, Y. 25th anniversary article: MXenes: a new family of two-dimensional materials. Advanced Materials, 26(7), 992-1005 (2014).

10. Hope, M. A., Forse, A. C., Griffith, K. J., Lukatskaya, M. R., Ghidiu, M., Gogotsi, Y., \& Grey, C. P. NMR reveals the surface functionalisation of Ti 3 C 2 MXene. Physical Chemistry Chemical Physics, 18(7), 5099-5102. (2016).

11. Feng, A., Yu, Y., Wang, Y., Jiang, F., Yu, Y., Mi, L., \& Song, L. Two-dimensional MXene Ti3C2 produced by exfoliation of Ti3AlC2. Materials \& Design, 114, 161-166. (2017).

12. Li, S., Zou, X., Xiong, X., Zheng, K., Lu, X., Zhou, Z., ... \& Xu, Q. Electrosynthesis of Ti3AlC2 from oxides/carbon precursor in molten calcium chloride. Journal of Alloys and Compounds, 735, 1901-1907. (2018).

13. Song, D., Jiang, X., Li, Y., Lu, X., Luan, S., Wang, Y., ... \& Gao, F. Metal- organic frameworks-derived $\mathrm{MnO} 2 / \mathrm{Mn} 3 \mathrm{O} 4$ microcuboids with hierarchically ordered nanosheets and Ti3C2 MXene/Au NPs composites for electrochemical pesticide detection. Journal of Hazardous Materials. (2019).

14. Liu, L., Zhao, Q., Liu, R., \& Zhu, L. Hydrogen adsorption-induced catalytic enhancement over $\mathrm{Cu}$ nanoparticles immobilized by layered Ti3C2 MXene. Applied Catalysis B: Environmental, 252, 198-204. (2019).

15. Chae, Y., Kim, S. J., Cho, S. Y., Choi, J., Maleski, K., Lee, B. J., ... \& Won, A. C. An investigation into the factors governing the oxidation of two-dimensional Ti3C2 MXene. Nanoscale. (2019).

16. Borysiuk, V. N., Mochalin, V. N., \& Gogotsi, Y. Molecular dynamic study of the mechanical properties of two-dimensional titanium carbides Tin+ $1 \mathrm{Cn}$ (MXenes). Nanotechnology, 26(26), 265705. (2015).

17. Maleski, K., Ren, C. E., Zhao, M. Q., Anasori, B., \& Gogotsi, Y. Size-dependent physical and electrochemical properties of two-dimensional MXene flakes. ACS applied materials \& interfaces, 10(29), 24491-24498. (2018).

18. Elmarakbi, A., Azoti, W. and Serry, M. Multiscale modelling of hybrid glass fibres reinforced graphene platelets polyamide PA6 matrix composites for crashworthiness applications. Applied Materials Today, 6, 1-8. (2017).

19. Vignesh, P., Kumar, R. K. and Ramu, M. Evaluation of Mechanical and Thermal Behaviour of Particle-Reinforced Metal Matrix Composite Using Representative Volume Element Approach. In Advances in Materials and Metallurgy, Springer, Singapore 415-425. (2019).

20. Zeleniakiene, D., Griskevicius, P., Norvydas, V., Aniskevich, A., \& Zukiene, K.. Simulation of mechanical behaviour of polychloroprene/versatic acid vinyl ester/methyl methacrylate/2-ethylhexyl acrylate copolymer blend. Iranian Polymer Journal, 27(2), 97 103. (2018).

21. Daunys, M., Dundulis, R., Kilikevičius, S., \& Česnavičius, R. Analytical investigation and numerical simulation of the stress-strain state in mechanically heterogeneous welded joints with a single-V butt weld. Engineering Failure Analysis, 62, 232-241. (2016).

22. Matveeva, A. Y., Lomov, S. V., \& Gorbatikh, L. Debonding at the fiber/matrix interface in carbon nanotube reinforced composites: Modelling investigation. Computational Materials Science, 159, 412-419. (2019). 
23. Hussein, A. and Kim, B. Micromechanics based FEM study on the mechanical properties and damage of epoxy reinforced with graphene based nanoplatelets. Composite Structures (2019).

24. Hussein, A. and Kim, B. Graphene/polymer nanocomposites: the active role of the matrix in stiffening mechanics. Composite Structures, 202, 170-181 (2018).

25. Pontefisso, A. and Mishnaevsky Jr, L. Nanomorphology of graphene and CNT reinforced polymer and its effect on damage: Micromechanical numerical study. Composites Part B: Engineering, 96, 338-349 (2016).

26. Dai, G. and Mishnaevsky Jr, L. Graphene reinforced nanocomposites: 3D simulation of damage and fracture. Computational Materials Science, 95, 684-692 (2014).

27. Eidel, B., Gote, A., Ruby, M., Holzer, L., Keller, L., \& Jiang, X. Estimating the effective elasticity properties of a diamond/ $\beta-\mathrm{SiC}$ composite thin film by $3 \mathrm{D}$ reconstruction and numerical homogenization. Diamond and Related Materials (2019).

28. Arora, G., \& Pathak, H. Modeling of transversely isotropic properties of CNT-polymer composites using meso-scale FEM approach. Composites Part B: Engineering, 166, 588597. (2019).

29. El Moumen, A., Tarfaoui, M., \& Lafdi, K. Computational homogenization of mechanical properties for laminate composites reinforced with thin film made of carbon nanotubes. Applied Composite Materials, 25(3), 569-588. (2018).

30. Hassanzadeh-Aghdam, M. K., Ansari, R., \& Darvizeh, A. Micromechanical analysis of carbon nanotube-coated fiber-reinforced hybrid composites. International Journal of Engineering Science, 130, 215-229. (2018).

31. Bahmani, A., Li, G., Willett, T. L., \& Montesano, J. (2019). Three-dimensional micromechanical assessment of bio-inspired composites with non-uniformly dispersed inclusions. Composite Structures, 212, 484-499.

32. Zhao, M. Q., et al. Flexible MXene/carbon nanotube composite paper with high volumetric capacitance. Advanced Materials, 27(2), 339-345 (2015).

33. Lee, C., Wei, X., Kysar, J. W. and Hone, J. Measurement of the elastic properties and intrinsic strength of monolayer graphene. Science, 321(5887), 385-388 (2008).

34. Lipatov, A., Lu, H., Alhabeb, M., Anasori, B., Gruverman, A., Gogotsi, Y. and Sinitskii, A. Elastic properties of 2D Ti3C2Tx MXene monolayers and bilayers. Science Advances, 4(6), eaat0491 (2018).

35. Jansson, S. Homogenized nonlinear constitutive properties and local stress concentrations for composites with periodic internal structure. International Journal of Solids and Structures, 29(17), 2181-2200. (1992). 